\title{
MODES OF ACCRETION IN X-RAY SOURCES
}

\author{
A. M. BELOBORODOV \\ Canadian Institute for Theoretical Astrophysics \\ University of Toronto, 60 St. George Street \\ Toronto, M5S $3 H 8$ Ontario, CANADA
}

\begin{abstract}
Three classical modes of accretion are briefly discussed: wind-fed, spherical, and disk. The three modes are illustrated with the mass transfer onto black holes in high-mass X-ray binaries. Then a new regime of mini-disk accretion is described and it is argued that observed wind-fed X-ray sources are likely to accrete in this regime. Switching from one accretion mode to another can cause the observed spectral state transitions.
\end{abstract}

\section{Introduction}

Accretion became an important subject of astrophysics 3 decades ago when it was realized to feed the brightest X-ray sources in the sky - X-ray binaries. These sources are associated with the most compact astrophysical objects: neutron stars and black holes. Accretion onto such objects has four major features: (1) It releases energy in a relativistically deep potential well, $\phi \sim-c^{2}$, and can have a high radiative efficiency. (2) It produces radiation in a small region of radius $r$ comparable to the Schwarzschild radius $r_{g}=2 G M / c^{2}=$ $0.3 \times 10^{6}\left(M / M_{\odot}\right)$ where $M$ is the object mass. (3) The observed luminosities $L$ are often comparable to the Eddington limit $L_{\mathrm{E}}=2 \pi r_{g} m_{p} c^{3} / \sigma_{\mathrm{T}} \approx 1.3 \times$ $10^{38}\left(M / M_{\odot}\right) \mathrm{erg} / \mathrm{s}$. (4) The high $L$ combined with the small size imply a high temperature of the source: a lower bound on the temperature can be estimated assuming blackbody emission, $k T_{\mathrm{bb}} \approx\left(L / \sigma r^{2}\right)^{1 / 4} \sim \mathrm{keV}$. This emission is in the X-ray band.

There are three textbook regimes of accretion: wind-fed (Hoyle \& Lyttleton 1 ; Bondi \& Hoyle 2), spherical (Bondi3), and disk (Shakura \& Sunyaev4). In all cases gas falls onto the accretor and releases its gravitational energy, yet the specific accretion mechanisms and the flow patterns differ. Here we briefly discuss these classical modes and illustrate them with black-hole accretion in high-mass X-ray binaries. Then we describe a new regime of mini-disk accretion.

\section{Wind-fed X-ray Binaries}

Mass transfer in an X-ray binary strongly depends on the type of the normal companion (donor). If the donor is massive (OB star) it emits a powerful wind 
which is partially captured by the compact companion. Thus, accretion occurs even if the donor does not fill its Roche lobe. We will focus on the windfed accretion here because it provides nice illustrations of different accretion modes. Also, historically, the first accretion scenario (Bondi-Hoyle-Lyttleton) was wind-fed.

The wind material is captured from a cylinder around the line that connects the two companions. It is called the accretion cylinder and its radius is

$$
R_{a}=\frac{2 G M}{w^{2}} \approx 3 \times 10^{10}\left(\frac{M}{M_{\odot}}\right)\left(\frac{w}{10^{8}}\right)^{-2} \mathrm{~cm},
$$

where $w$ is the wind velocity and $M$ is the mass of the accretor. Winds from massive OB stars have $w \approx 10^{8} \mathrm{~cm} \mathrm{~s}^{-1}$, approximately equal to the escape velocity from the star surface. The separation of the observed X-ray binaries, $A \sim 10^{12} \mathrm{~cm}$, is much larger than $R_{a}$. This has two consequences: (1) only a small fraction $\sim\left(R_{a} / A\right)^{2}$ of the wind is captured and (2) the flow inside the accretion cylinder is nearly plane-parallel before it gets trapped by the gravitational field of the accretor.

The wind crosses the distance $A$ and accretes on a timescale $A / w \sim 10^{4} \mathrm{~s}$. It is much shorter than the orbital period of the binary, $P \sim 10^{5}-10^{6} \mathrm{~s}$ and one could expect that the orbital rotation weakly affects the accretion flow; in particular, the net angular momentum $\bar{l}$ of the trapped gas must be close to zero. In fact, even a slow orbital rotation and a corresponding small $\bar{l} \neq 0$ can strongly impact the mechanism of accretion: the trapped gas falls many decades in radius onto the compact object $\left(R_{a} / r_{g} \approx 10^{5}\right)$ and the approximation $\bar{l}=0$, which is good at $R \sim R_{a}$, can fail before the gas reaches $r_{g}$. A very small $\bar{l} \sim r_{g} c$ would be sufficient for a disk formation near the compact object.

The trapped $\bar{l}$ was estimated and compared with $r_{g} c$ by Illarionov \& Sunyaev 6 and Shapiro \& Lightman 6 . Consider the accretion cylinder as a sequence of moving pancakes of radius $R_{a}$ (perpendicular slices of the cylinder). When viewed from the accretor, the pancakes have the orbital angular velocity $\Omega=2 \pi / P$ and their specific angular momentum is $\bar{l}=(1 / 4) \Omega R_{a}^{2}$. Using equation (1) one gets $\bar{l} /\left(r_{g} c\right) \approx(P / \text { day })^{-1}\left(M / \mathrm{M}_{\odot}\right)\left(w / 10^{8}\right)^{-4}$. Three X-ray binaries are classified as massive ones with black hole companions: Cyg X-1, LMC X-1, and LMC X-3 (see Tanaka \& Lewin). They have orbital periods $P=5.6,4.2$, and $1.7 \mathrm{~d}$, respectively, and masses $M$ of order of $10 M_{\odot}$. One thus obtains $\bar{l} \sim r_{g} c$ for these objects, which is marginal for formation of a small-scale disk. Whether the disk forms or not depends on the precise value of $\bar{l}$ which is difficult to calculate because the details of wind accretion are not fully understood (note the strong dependence of $\bar{l}$ on the wind velocity, $w$ ). 


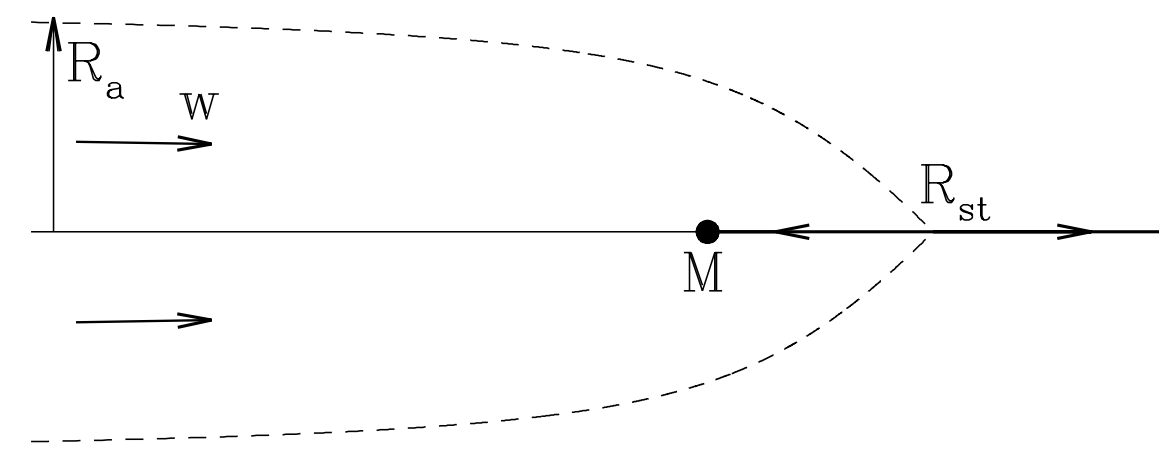

Figure 1: Bondi-Hoyle-Lyttleton accretion. An initially plane-parallel flow is deflected by the accretor and focused onto the axis of symmetry (the accretion line). Further accretion proceeds along the line inside a stagnation radius $R_{\mathrm{st}}$.

At distances $R \gg r_{g}$ from the accretor one can assume $\bar{l}=0$ and we first discuss models with zero net angular momentum (Sect. 3 and 4 ).

\section{Bondi-Hoyle-Lyttleton Accretion}

The original model of Bondi \& Hoyle日 envisions an initially plane-parallel flow interacting with an accretor of mass $M$ (Fig. 1). The accretor deflects the gas streamlines from the straight lines and captures a part of the flow. The model neglects the gas pressure, so the streamlines follow ballistic (free-fall) trajectories of test particles in the gravitational field of the accretor. The problem has a symmetry axis and the deflected trajectories intersect on the axis behind the accretor. This one-dimensional caustic (called the accretion line) is assumed to be sticky. The gas in the caustic has a velocity $u^{r}$ which differ from the the radial component $\hat{u}^{r}$ of the free fall that impinges on the caustic. The gas motion in the caustic obeys the momentum conservation law,

$$
\frac{\mathrm{d} u^{r}}{\mathrm{~d} r}=\frac{\mathrm{d} \dot{M}}{\mathrm{~d} r} \frac{\left(\hat{u}^{r}-u^{r}\right)}{\dot{M}(r)}-\frac{G M}{r^{2} u^{r}} .
$$

Here $\dot{M}(r)$ is the flux of mass in the caustic, and $\mathrm{d} \dot{M} / \mathrm{d} r$ is its feeding rate, which is easily calculated given the ballistic trajectories of the infall. The infall momentum is directed away from the accretor and its inertia competes with gravity. Gravity dominates at small $r$ and here $u^{r}<0$ (gas flows in) while at large radii $u^{r}>0$ (gas flows out). Hence there must be a stagnation 
point on the line at a radius $R_{\mathrm{st}} \sim R_{a}$. The $R_{\mathrm{st}}$ is not well defined in the model — infinite number of steady solutions with different $R_{\text {st }}$ exist. For a given solution $u^{r}(r)$ one easily calculates the dissipated energy: $\mathrm{d} E_{\mathrm{diss}} / \mathrm{d} r=$ $(\mathrm{d} \dot{M} / \mathrm{d} r)\left[\hat{u}_{\perp}^{2}+\left(\hat{u}^{r}-u^{r}\right)^{2}\right] / 2$ where $\hat{u}_{\perp}$ is the non-radial velocity component that is canceled where the ballistic flow reaches the caustic.

This simple and beautiful model does not, however, apply to real X-ray sources. The model is based on the assumption that the colliding gas on the accretion line immediately emits the dissipated energy. In fact, the radiative losses are small on the $R_{a} / w$ timescale and the stored heat creates a high pressure that forces the shocked gas to expand away from the caustic. As a result a steady bow shock sets in at a distance $\sim R_{a}$ in front of the accretor. Hunt first studied the subsonic gas dynamics behind the shock and showed that a spherically symmetric inflow forms at distances $R<{ }_{0} R_{a}$ from the accretor. Subsequent numerical simulations (e.g. Blondin et al. \%; Ruffert 10.11 ) showed that the flow is unstable and variable if the bow shock is strong, i.e. if its Mach number $\mathcal{M}=w / c_{s}$ is well above unity [here $c_{s}=\left(10 \mathrm{kT} / 3 m_{p}\right)^{1 / 2}$ is the upstream sound speed]. In the case of a modest $\mathcal{M} \gtrsim 1$ the variations are weak and the flow is approximately laminar. This is the most likely case if accretion occurs in the radiation field of a luminous X-ray source which preheats the upstream flow (Illarionov \& Beloborodov 12 ). The laminar transformation of an initially plane-parallel flow into a spherical infall is schematically shown in Fig. 2.

\section{Spherical Accretion}

The first detailed study of spherically symmetric (radial) accretion was done by Bondi 3 . The steady gas dynamics is governed by simple equations of baryon and momentum conservation,

$$
4 \pi R^{2} \rho u=\dot{M}=\text { const, } \quad u \frac{\mathrm{d} u}{\mathrm{~d} R}=-\frac{1}{\rho} \frac{\mathrm{d} p}{\mathrm{~d} R}-\frac{G M}{R^{2}} .
$$

The problem has three unknowns: baryon density $\rho$, pressure $p$, and accretion velocity $u$. Hence one more equation is needed. In the Bondi model the set of equations is closed by assuming a polytropic equation of state $p=K \rho^{\Gamma}$. One can then find a family of solutions specified by an accretion rate $\dot{M}$ and an inner boundary condition. The inner boundary differs for neutron stars and black holes; in the latter case the flow must pass through a sonic point since the gas supersonically falls in at the event horizon. The standard theory of polytropic sphericalaccretion is explained in details in textbooks (see e.g. Shapiro \& Teukolsky 13; Frank, King \& Raine 14). 


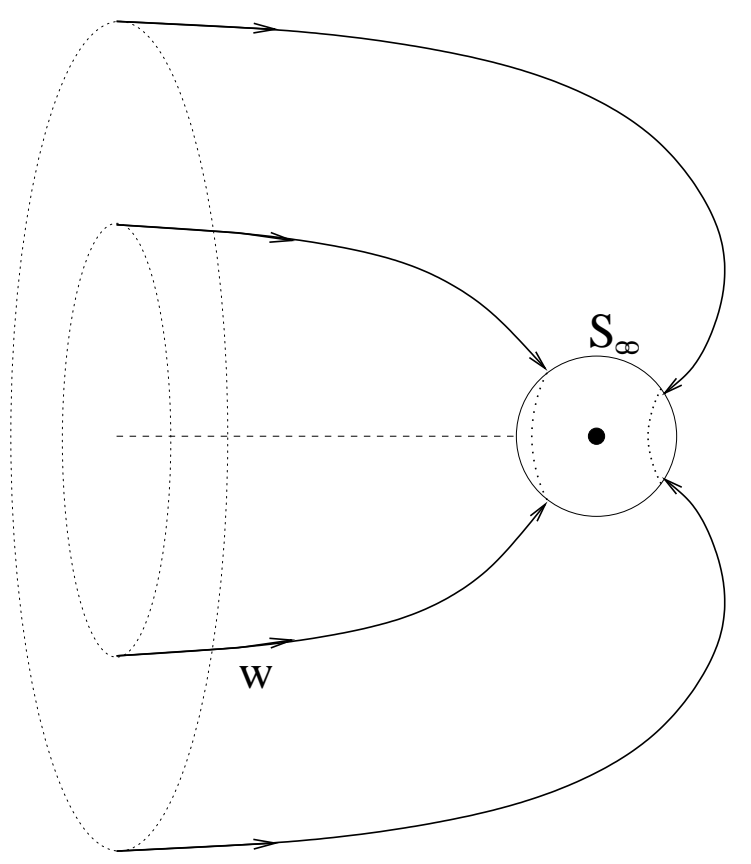

Figure 2: Transformation of a plane-parallel flow into a spherical infall. $S_{\infty}$ denotes the sphere of radius $R \sim R_{\mathrm{C}}$ (eq. 6). Inside $S_{\infty}$, the flow is Compton cooled and falling freely.

This theory, however, does not apply to luminous X-ray sources, as was pointed out by Zel'dovich \& Shakura 15 . The accreting gas exchanges energy with the radiation field of the source through Compton scattering and the effective $\Gamma \neq$ const. Compton scattering dominates over other radiative processes and the thermal balance of the accreting gas reads

$$
\frac{\mathrm{d} T}{\mathrm{~d} t}=-\frac{2}{3} T \operatorname{div} \mathbf{v}+\frac{T_{\mathrm{C}}-T}{t_{\mathrm{C}}} .
$$

The first term on the right-hand side is the compressive heating, and the second term describes the energy exchange with the radiation on the Compton timescale $t_{\mathrm{C}}=3 \pi m_{e} c^{2} R^{2} /\left(\sigma_{\mathrm{T}} L\right)$. The $T_{\mathrm{C}}$ is the Compton temperature of the radiation field. If the radiation scatters off a plasma with $T>T_{\mathrm{C}}$, the photons on average gain energy i.e. cool the plasma, while at $T<T_{\mathrm{C}}$ the photons loose energy (because of the quantum recoil effect in Compton scattering) and heat the plasma. The $T_{\mathrm{C}}$ is defined so that a plasma with $T=T_{\mathrm{C}}$ is in energy 
equilibrium with the radiation field. It is determined by the shape of the radiation spectrum $F_{\nu}$,

$$
k T_{\mathrm{C}}=\frac{\int F_{\nu} h \nu \mathrm{d} \nu}{4 \int F_{\nu} \mathrm{d} \nu} .
$$

In hard X-ray sources $T_{\mathrm{C}} \sim 10^{8} \mathrm{~K}$.

The temperature of the wind far from the accretor is relatively low, $T_{0} \sim$ $10^{5} \mathrm{~K} \ll T_{\mathrm{C}}$, and where the flow approaches the X-ray source it is heated by Compton scattering. Inside $R_{a}$, just where the spherical inflow is forming, the gas is still heated, so that the effective $\Gamma>5 / 3$ and changes with $R$. Compton heating strongly affects the flow, breaks the spherical symmetry, and leads to formation of an inflow-outflow pattern of accretion (Ostriker et al. 6 ; Illarionov \& Kompaneets 17; Igumenshchev, Illarionov, \& Kompaneets 18 ). The situation changes inward of the so-called Compton radius

$$
R_{\mathrm{C}}=\frac{G M m_{p}}{5 k T_{\mathrm{C}}} \approx 0.1 R_{a} .
$$

Here the escape temperature $T_{\text {esc }}(R)=G M m_{p} /(5 k R)$ exceeds $T_{\mathrm{C}}$ and a stable spherical inflow forms with $\mathcal{M}>1$. The plasma is now cooled by the X-rays rather than heated. The cooling timescale $t_{\mathrm{C}}$ gets shorter than the compressive heating timescale $R / v$, and the gas is cooled well below $T_{\text {esc }}$. It implies that the pressure term in the momentum equation (3) is small compared to the gravitational term and gas falls almost freely, with a high Mach number. Magnetic fields trapped from the donor wind are amplified and dissipated in the converging infall (Bisnovatyi-Kogan \& Ruzmaikin19; Mészáros 20). Their energy should not, however, exceed the gas thermal energy, and therefore the fields should not affect the free-fall.

The Compton-cooled radial free-fall at $R \ll R_{\mathrm{C}}$ emits little radiation. Its low pressure implies that the compressive heating is weak and the gravitational energy of the gas transforms largerly into the ballistic kinetic energy. If the compact object is a black hole, this energy is swallowed along with the flow, leading to a low radiative efficiency of accretion. This regime can hardly take place in the observed bright sources like Cyg X-1. An appropriate mode of accretion for these sources should be able to dissipate efficiently the infall energy. Dissipation can naturally occur if the gas has a non-zero angular momentum and forms a disk before plunging into the event horizon. We now turn to accretion with non-zero angular momentum. 


\section{Viscous Disk}

Accretion with angular momentum $l$ is stopped by the centrifugal barrier at a radius $r=l^{2} / G M$. Here the gas forms a ring that rotates at about Keplerian angular velocity $\Omega_{\mathrm{K}}=\left(G M / r^{3}\right)^{1 / 2}$. In this section we focus on models with $l \gg r_{g} c$. This is the condition of the standard disk model where steady accretion is possible only if some mechanism redistributes angular momentum and allows the gas to spiral toward the centre. A plausible viscosity mechanism is provided by MHD turbulence that develops due to the differential character of Keplerian rotation (see Balbus \& Hawley 11 for a review). Viscosity dissipates the orbital energy of the rotating gas and the gas diffuses to more bound circular orbits. A steady accretion disk dissipates energy with a rate $F_{\text {diss }}$ per unit area,

$$
F_{\text {diss }}(r)=\frac{3 \dot{M}}{4 \pi} \Omega_{\mathrm{K}}^{2} S,
$$

where $S(r)$ is a numerical factor determined by the inner boundary condition. For a Schwarzschild black hole $S=1-\left(3 r_{g} / R\right)^{1 / 2}$ so that $S=0$ at the inner boundary of the viscous disk, $r=3 r_{g}$, where the gas starts to fall freely into the hole. This standard disk model was successfully applied to X-ray binaries and active galactic nuclei (AGN), the latter being just a scaled version of the standard model that corresponds to a massive black hole, $M=10^{6}-10^{9} M_{\odot}$.

If the dissipated energy is immediately radiated away, the emerging radiation flux from the disk surface is $F(r)=F_{\text {diss }}(r) / 2$ (the factor $1 / 2$ accounts for the two faces of the disk). The total emitted flux is easily calculated, however, its spectrum $F_{\nu}$ is difficult to predict. The simplest model assumes that the disk emits as a black body and then one finds the radial profile of its surface temperature, $T_{\mathrm{bb}}=(F / \sigma)^{1 / 4} \propto r^{-3 / 4} S^{1 / 4}$. The integrated disk spectrum (multicolor blackbody) has an exponential cutoff at $\sim \mathrm{keV}$ in X-ray binaries and $\sim 10 \mathrm{eV}$ in AGN. Such emission (with the right temperature) is indeed observed in many sources. However, in addition, one observes a second spectral component that peaks at about $100 \mathrm{keV}$. Example spectra of the famous black hole candidate Cyg X-1 are shown in Fig. 3. Most of the time this object spends in the 'hard' state when the $100 \mathrm{keV}$ component is dominant. Sometimes it switches to the 'soft' state where the hard emission is weak and the soft blackbody component is strong.

Phenomenologically, a two-peak emission spectrum is well explained. There must be two gaseous components that are heated in the accretion flow: one, dense, emits as a black body (presumably an optically thick accretion disk), and the other one is rarefied and hot, with $k T \sim 100 \mathrm{keV}$. A fraction of black-

body photons from the dense component get upscattered in the hot plasma, 


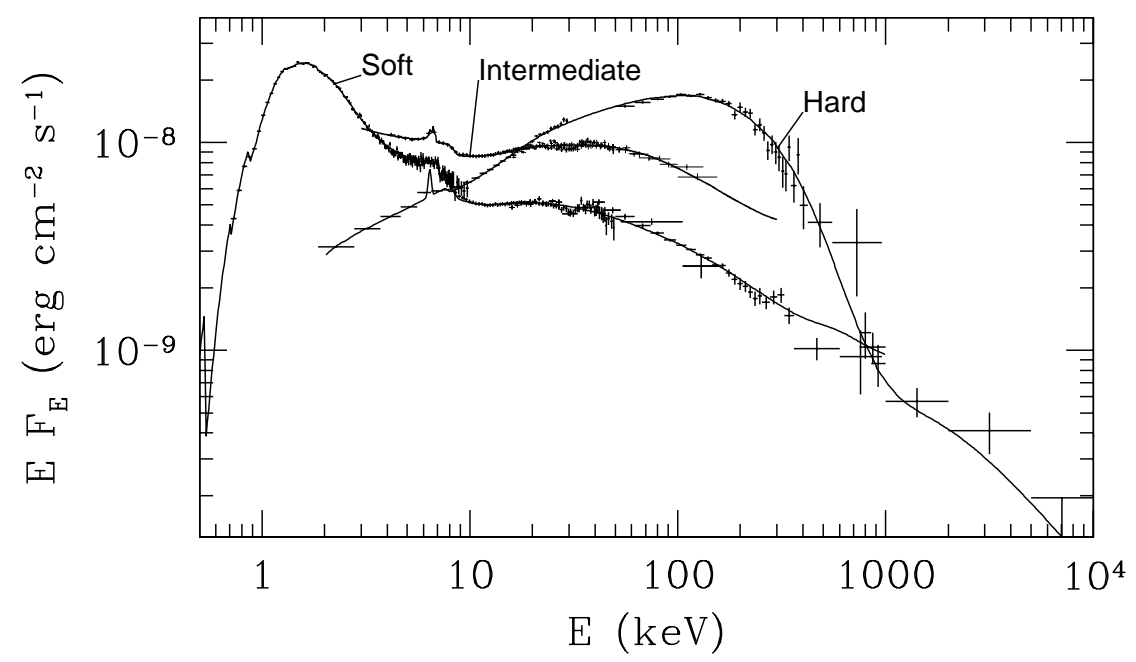

Figure 3: Spectrum of Cyg X-1 in the hard, soft, and intermediate states. The hardstate spectrum is obtained in 1991 by CGRO (Gierliński et al. ${ }^{22}$. McConnell et al. ${ }^{23}$. The intermediate-state spectrum was obtained by RXTE on May 23, 1996. The soft-state spectrum was obtained by ASCA and RXTE on May 30, 1996 and by OSSE between June 14-25, 1996 (Gierliński et al. ${ }^{24}$ ). The solid curves show the best fits to the data by the EQPAIR models. (From Coppi ${ }^{24}$.)

become hard X-rays, and form the second spectral peak (e.g. Poutanen 26, Zdziarski 27). This Comptonization process is well understood and one can derive the optical depth $\tau_{\mathrm{T}}$ (to electron scattering) of the hot plasma from the spectral data. Interestingly, one finds $\tau_{\mathrm{T}} \approx 1$ and $k T \approx 100 \mathrm{keV}$ in various sources which include both X-ray binaries and AGN. However the geometry of the hot+cold gas is still unknown and it is unclear why the accretion flow chooses to heat gas with $\tau_{\mathrm{T}} \approx 1$. Two basic suggestions are: (1) buoyant magnetic loops are generated in the turbulent disk and form an active corona (Galeev, Rosner \& Vaiana 28 ), and (2) the cold disk is transformed into a hot two-temperature torus at a radius $r_{\mathrm{tr}}$; the electron temperature in the torus is $k T_{e} \approx 100 \mathrm{keV}$ (Shapiro, Lightman \& Eardley 29). The two modifications of the standard disk look likely (see Beloborodov 30 for a review), yet both are strongly dependent on the poorly understood viscosity/heating mechanism.

At small accretion rates, the viscous disk may switch to the advective regime where the released heat is transported into the hole before it can be radiated away. The hot advective disks have low luminosities, much below $\dot{M} c^{2}$, and can apply to weak X-ray sources (Menou, Blandford, these proceedings). 


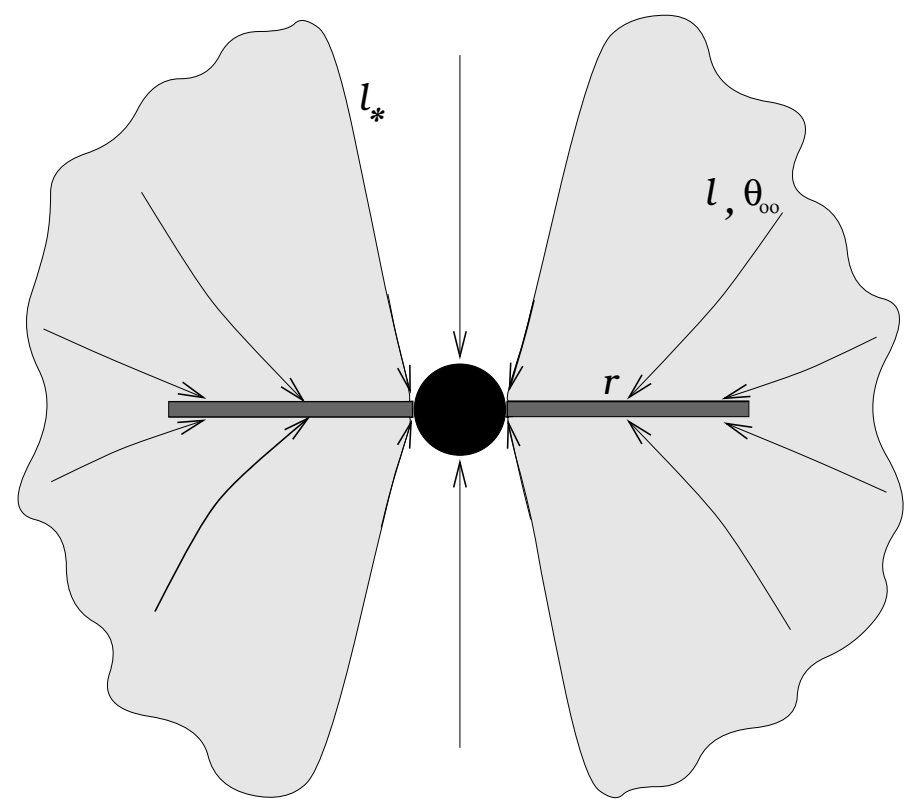

Figure 4: Schematic picture of the disk formation. The inflow has angular momentum $l\left(\theta_{\infty}\right)$ increasing toward the equatorial plane, $\mathrm{d} l / \mathrm{d} \sin \theta_{\infty}>0$. The collision radius, which is a growing function of $l$, monotonically increases as $\theta_{\infty}$ approaches $\pi / 2$. The shadowed parts of the accretion flow collide outside $r_{g}$ and form a couple of radiative shocks which sandwich the caustic. The other part of the flow (at small polar angles where $l<l_{*}$ ) is directly swallowed by the black hole with a low radiative efficiency.

\section{Mini-Disk}

As it was discussed in Sect. 2, the estimated angular momentum of the windfed accretion flows in X-ray binaries is marginally enough to form a disk. Is a disk actually formed there? - Probably yes, because otherwise accretion would occur in the quasi-spherical regime with little dissipation, which is hard to reconcile with the observed high luminosities. If so, at what radius $r_{0}$ does the disk form and how does it compare with $3 r_{g}$ (the inner edge of the standard disk)? What is the critical radius $r_{\mathrm{cr}}$ for the viscous disk formation?

The critical radius turns out quite large, $r_{\mathrm{cr}} \approx 14 r_{g}$, and the accretion regime with $r_{g}<r_{0}<r_{\text {cr }}$ needs to be addressed (Beloborodov \& Illarionov 11 ). Such a 'mini-disk' is very different from the standard viscous disk as regards both the dynamics and the emission mechanism. 


\subsection{The Caustic}

Fig. 4 schematically shows the mini-disk formation in the inner region of a quasi-spherical inflow with angular momentum. The inflow is almost radial at large radii, it is Compton cooled at $R_{\mathrm{C}} \sim 10^{4} r_{g}$ and falls freely toward the center. Near the black hole, the rotation deflects the infall from the radial direction and a disk-like caustic appears in the defocused flow. The flow is assumed to be symmetric around the spin axis and also symmetric about the equatorial plane. In this plane a ballistic streamline collides with its symmetric counterpart. The radius of collision is determined by the angular momentum of the streamline, $l$,

$$
r=\frac{l^{2}}{G M}-\frac{r_{g}}{7}
$$

where $r_{g} / 7$ is a relativistic correction to the Newtonian formula. The angular momentum of a streamline depends on its initial polar angle at $R \gg r_{g}$, which we denote $\theta_{\infty}$. A likely distribution $l\left(\theta_{\infty}\right)$ has $l=0$ on the polar axis $\left(\theta_{\infty}=\right.$ $0, \pi)$ and a maximum $l_{0}$ at the equatorial plane $\left(\theta_{\infty}=\pi / 2\right)$. A simple example is a 'solid body' rotation, $l\left(\theta_{\infty}\right)=l_{0} \sin \theta_{\infty}$. From equation (8) one can see that streamlines with larger $l$ collide at larger $r$. The polar part of the inflow with $l<l_{*}=0.75 r_{g} c$ crosses the event horizon before it can collide, while the equatorial part with $l>l_{*}$ forms a caustic outside $r_{g}$ (Fig. (1). The outer edge of the caustic is defined by the streamlines with $\theta_{\infty} \rightarrow \pi / 2$ and its radius is $r_{0}=l_{0}^{2} / G M-r_{g} / 7$. The condition for the disk formation outside the black hole $\left(r_{0}>r_{g}\right)$ reads $l_{0}>l_{*}$.

When the ballistic flow approaches the caustic, it passes through a shock and then continues to accrete through the disk. A collisionless shock forms on

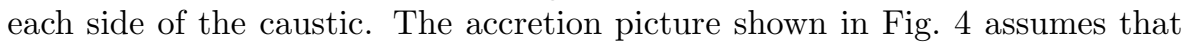
the shock is radiative and pinned to the equatorial plane. This is indeed the case if the source luminosity exceeds $10^{-2} L_{\mathrm{E}}$ (Beloborodov \& Illarionov 31 ). The shocked ions pass their energy via Coulomb collisions to the radiatively efficient electrons on a timescale

$$
t_{\mathrm{Coul}}=\frac{\sqrt{\pi / 2} m_{p} \theta_{e}^{3 / 2}}{m_{e} c \sigma_{\mathrm{T}} z n \ln \Lambda} \approx 17 \frac{T_{e}^{3 / 2}}{z n} \mathrm{~s}, \quad \theta_{e} \equiv \frac{k T_{e}}{m_{e} c^{2}} .
$$

Here $n$ is the postshock proton density, $\ln \Lambda \approx 15$ is a Coulomb logarithm, and $z>1$ accounts for the possible $e^{ \pm}$production. The postshock electrons have a much smaller temperature than the protons, $T_{e} \ll T_{p}$. They immediately radiate the received heat by Compton scattering, and $T_{e}$ is controlled by the cooling=heating balance,

$$
\frac{T_{e}}{t_{\mathrm{C}}}=\frac{T_{p}}{t_{\mathrm{Coul}}}
$$


where $t_{\mathrm{C}}=3 m_{e} c^{2} /\left(8 \sigma_{\mathrm{T}} F\right)$ is the Compton cooling time-scale and $F$ is the local radiation flux. For a radiative shock $F \sim(3 / 2) n k T_{p} v_{\mathrm{ff}} / \xi$ where $v_{\mathrm{ff}}=$ $(2 G M / r)^{1 / 2}$ is the free-fall velocity at a radius $r$ and $\xi \geq 4$ is the compression at the shock front. Then $t_{\mathrm{C}} \approx m_{e} c^{2} \xi /\left(8 \sigma_{\mathrm{T}} n k T_{p} v_{\mathrm{ff}}\right)$ and the energy balance (10) yields

$$
\frac{k T_{e}}{m_{e} c^{2}} \approx\left(\frac{\ln \Lambda m_{e}}{\sqrt{2 \pi} m_{p}} \frac{\xi c}{4 v_{\mathrm{ff}}}\right)^{2 / 5} \approx 0.1\left(\frac{\xi c}{4 v_{\mathrm{ff}}}\right)^{2 / 5} .
$$

Density scaled out from this estimate. Given $\xi \approx 4$ and $v_{\mathrm{ff}} \approx c$ one finds $k T \approx 100 \mathrm{keV}$.

The hot postshock layer that radiates most of the proton heat has optical depth $\tau_{\mathrm{T}} \approx\left(v_{\mathrm{ff}} / \xi\right) t_{\mathrm{Coul}} z n \sigma_{\mathrm{T}}$ and satisfies the relation $\tau_{\mathrm{T}} \theta_{e} \approx 0.1$; one thus gets $\tau_{\mathrm{T}} \sim 1$ for typical $\theta_{e} \sim 0.1$. In deeper layers, at $\tau_{\mathrm{T}}>1, T_{p}$ and $T_{e}$ fall off sharply and the shocked plasma condenses to a thin dense disk. A similar radiative shock on a surface of a peutron star was studied by Zel'dovich \& Shakura 15 and Shapiro \& Salpeter 32.

The pattern of accretion with shocks pinned to the caustic requires a sufficiently high accretion rate $\dot{M}$ corresponding to the disk luminosity $L>$ $10^{-2} L_{\mathrm{E}}$. At smaller $\dot{M}$ the shocked plasma is unable to cool rapidly and it supports a quasi-spherical shock front far away from the disk. Such a flow was simulated numerically by Igumenshchev, Illarionov \& Abramowicz 33 . The simulations show that the shock tends to shrink toward the disk of radius $l_{0}^{2} / G M$ when $\dot{M}$ increases.

\subsection{Accretion in the Mini-Disk}

The disk is composed of the shocked matter that entered the disk with different horizontal velocities. A strong turbulence develops under such conditions and efficiently mixes the disk in the vertical direction. As a result, the disk shares the horizontal momentum with the feeding infall and accretes with a verticallyaveraged horizontal velocity. This situation can be described as a 'sticky' caustic, and it resembles the accretion line in the Bondi-Hoyle-Lyttleton (BHL) problem. The infall absorption by the sticky caustic implies energy dissipation, and the released heat is radiated away, contributing to the total luminosity. The process of disk-infall inelastic collision is governed by the corresponding law of momentum conservation. The relativistic conservation law $\nabla_{k} T_{i}^{k}=0$ in the Schwarzschild metric yields

$$
\frac{\mathrm{d} u^{r}}{\mathrm{~d} r}=\frac{\mathrm{d} \dot{M}}{\mathrm{~d} r} \frac{\left(\hat{u}^{r}-u^{r}\right)}{\dot{M}(r)}-\frac{G M}{r^{2} u^{r}}\left(1-\frac{j^{2}}{j_{\mathrm{K}}^{2}}\right)
$$




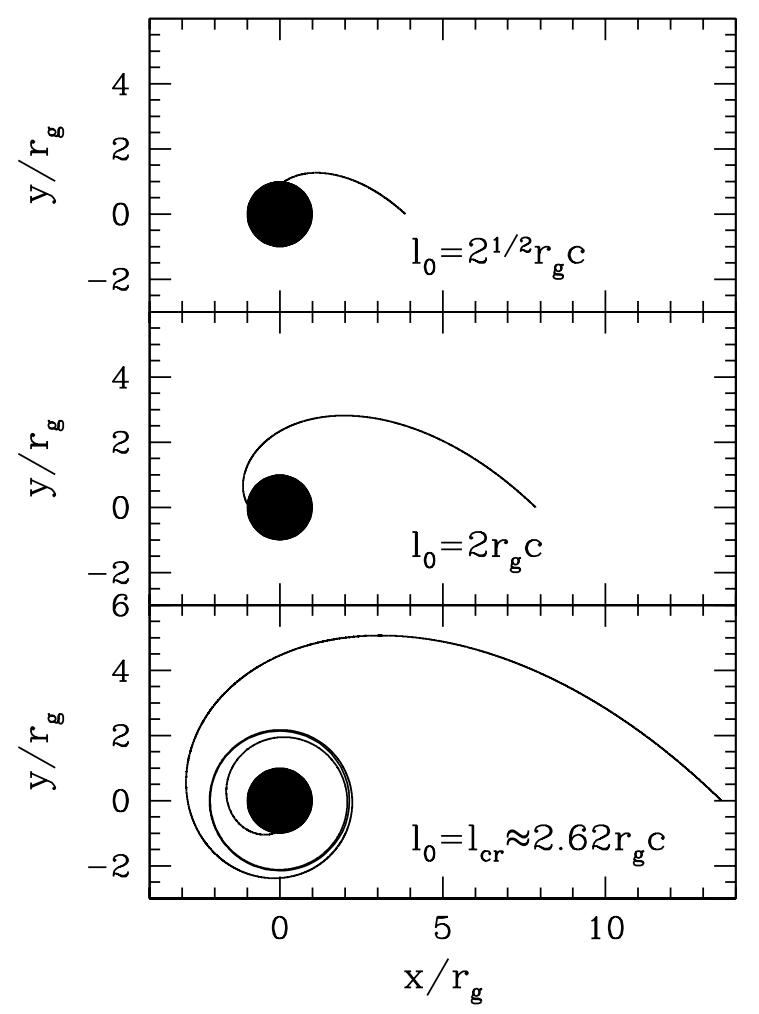

Figure 5: The trajectory of gas motion in the disk, from the outer edge into the black hole. In the critical case (bottom panel) the trajectory makes an infinite number of revolutions at the critical radius $r_{\mathrm{cr}} \approx 2.14 r_{g}$ before falling into the black hole.

where $j_{\mathrm{K}}^{2}(r)=r^{2} r_{g} c^{2} /\left(2 r-3 r_{g}\right)$ is the squared angular momentum of circular Keplerian orbits. The first term on the right-hand-side is a result of the disk-infall interaction. Equation (12) is similar to the BHL equation (2) that describes gas motion along the accretion line. The only difference is the additional term proportional to $j^{2} / j_{\mathrm{K}}^{2}$. It appears because now the accreting matter rotates and experiences a centrifugal acceleration. The repulsive centrifugal force dominates over gravity if $j>j_{\mathrm{K}}$ and it can stop accretion. Therefore, there exists a critical angular momentum $j_{\text {cr }}$ above which the freefall accretion is stuck and requires viscosity like the standard disk does.

The critical angular momentum can be calculated. For illustration, consider a flow with $l\left(\theta_{\infty}\right)=l_{0} \sin \theta_{\infty} ; l_{0}$ is the only parameter of the problem. 


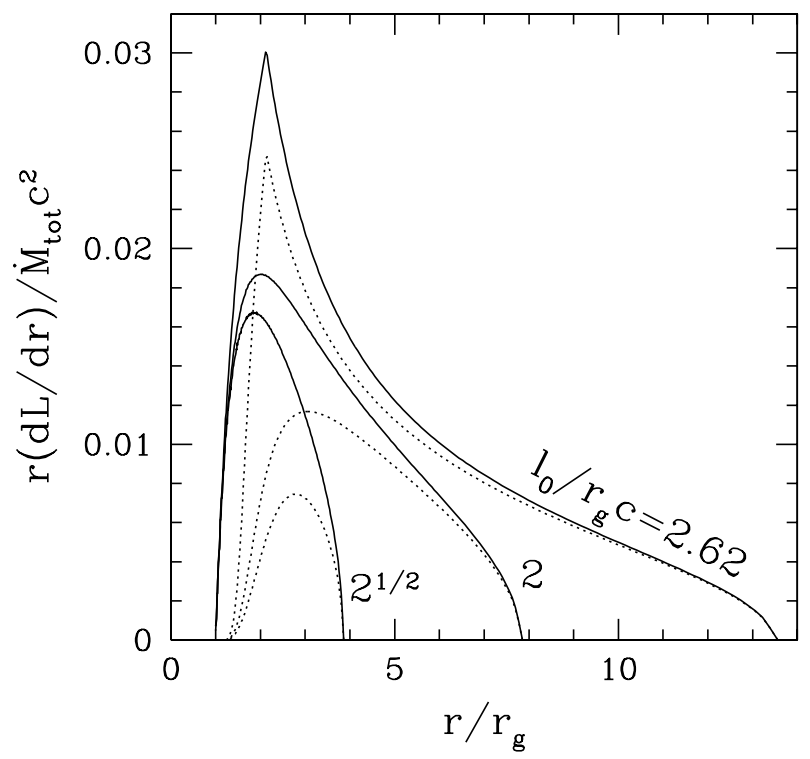

Figure 6: The radial distribution of the disk luminosity: solid curves - the released luminosity, dotted curves - the observed luminosity corrected for the radiation capture by the black hole. Three models are shown with $l_{0}=j_{\mathrm{cr}} \approx 2.62 r_{g} c, l_{0}=2 r_{g} c$, and $l_{0}=\sqrt{2} r_{g} c$.

The calculated streamlines of the mini-disk are shown in Fig. 5. A disk with $l_{0}<j_{\mathrm{cr}}=2.62 r_{g} c$ accretes on the free-fall timescale, with negligible horizontal viscous stresses. Its radius can be as large as $14 r_{g}$. A streamline of the disk starts at the outer edge $r_{0}$ with a relatively large angular momentum $j=l_{0}$ and then, as the streamline approaches the black hole, $j$ decreases. This happens because at smaller $r$ the disk absorbs the infall with smaller angular momentum; it allows the disk to overcome the centrifugal barrier without horizontal viscous stresses and accrete even with an initially large $l_{0}$. The bottleneck for accretion is at $2 r_{g}$ and here accretion can occur only if $j<2 r_{g} c$. One can thus derive a necessary condition for the inviscid accretion regime that reads $\bar{l}<2 r_{g} c$ where $\bar{l}$ is the mean angular momentum of the whole flow.

\subsection{Emission from the Mini-Disk}

The energy dissipated in the inelastic disk-infall interaction is easily calculated for a given dynamic solution, like it was done in the BHL model. The radial 
distribution of the resulting luminosity is shown in Fig. 6 . It peaks at $2 r_{g}$ where the centrifugal deceleration of the inflow is strongest (the bottleneck for accretion). Here the relative velocity between the disk and the infall increases and the inelastic collision liberates more energy. The small size of the main emitting region implies that the released luminosity is partially captured into the black hole, and the corresponding correction is shown in Fig. 6 .

The net radiative efficiency of accretion $\eta=L / \dot{M} c^{2}$ depends on $l_{0}$ : it rises from $\eta=0$ at $l_{0}<l_{*}$ to a maximum $\eta \approx 0.0372$ at $l_{0}=j_{\mathrm{cr}}$. This maximum is comparable to the efficiency of the standard disk around a Schwarzschild black hole, $\eta=1-2 \sqrt{2} / 3 \approx 0.0572$.

The energy is released in two steps: (1) at the shock front where the vertical momentum of the infall is canceled and (2) inside the disk where the infall inelastically shares its horizontal momentum with the disk. The first step heats a postshock layer with $\tau_{\mathrm{T}} \approx 1$ and $k T_{e} \approx 100 \mathrm{keV}$, and the subsequent dissipation in the geometrically thin dense disk can emit optically thick cool radiation. The two gaseous components thus emit a two-peak radiation spectrum that is typical for black-hole sources.

\subsection{Comparison with Other Accretion Models}

The mini-disk is a sticky caustic in a freely falling flow and in this respect it resembles the BHL accretion. In their case, accretion proceeds along a onedimensional caustic, the accretion line. The accretion line is fed at each point by matter from an initially plane-parallel flow which is axisymmetric and has zero net angular momentum. The mini-disk is a two-dimensional caustic in an asymptotically isotropic inflow with a non-zero net angular momentum. In contrast to the BHL accretion line, the radial momentum of matter impinging on the disk is directed inward. As a result, the mini-disk does not have the degenerate stagnation radius that exists in the BHL problem.

For large angular momenta, the disk accretion is stopped by the centrifugal barrier and then the mini-disk regime switches to the standard viscous regime. The major differences of the mini-disk from its standard counterpart are: (1) it is inviscid and accretes supersonically, (2) the energy release in the disk is caused by the infall absorption, and (3) most of the energy is released inside $3 r_{g}$.

\section{Discussion}

Angular momentum $l$ is a major parameter of an accretion flow. Two classical modes of accretion - spherical inflow and viscous disk - are valid in the limits $l \ll r_{g} c$ and $l \gg r_{g} c$, respectively. The two limits are bridged by an 
intermediate, but qualitatively different, mini-disk regime. It takes place for the range of the disk radii $r_{g}<r_{0}<14 r_{g}=28 G M / c^{2}$.

Motivation for the theoretical study of the mini-disk comes from the observed high-mass X-ray binaries with black holes: the wind-fed accretion flows there have $l \sim r_{g} c$. This fact is interesting: why should wind-fed black holes accrete near the threshold for disk formation? One possible explanation is as follows. Suppose that the dominant majority of black holes with massive companions accrete gas with small $l<l_{*}$. It should be difficult to observe such objects because of their low luminosity. When $l$ exceeds $l_{*}$ and the disk forms, the luminosity rises dramatically and the black hole switches on as an $\mathrm{X}$-ray source. One therefore expects to see preferentially objects with $l$ above the threshold for disk formation. If the $l$-distribution of objects falls steeply toward high $l$, most of the bright sources should be near the threshold, i.e. the regime $\bar{l} \sim r_{g} c$ can be widespread among observed bright sources.

One of the most intriguing properties of accreting black holes is their spectral state transitions. An example of such transitions in Cyg X-1 is shown in Fig. 3. The transitions may be caused by modest changes of the angular momentum of the accretion flow. Such changes should naturally occur since the trapped $l$ is very small and sensitive to the fluctuations of the donor wind. Suppose a black hole accretes in the mini-disk regime and emits a strong $100 \mathrm{keV}$ emission. If the angular momentum increases by a factor of 2 , the disk has to switch to the standard viscous regime with a higher luminosity and softer spectrum. This results in a hard-to-soft transition. If the angular momentum decreases by a factor of 2 , the source luminosity would fall down. Similar

spectral state transitions were recently observed in LMC X-3 (Wilms et al.34).

\section{References}

1. F. Hoyle and R.A. Lyttleton, Proc. Cam. Phil. Soc. 35, 405 (1939).

2. H. Bondi and F. Hoyle, MNRAS 104, 273 (1944).

3. H. Bondi, MNRAS 112, 195 (1952).

4. N.I. Shakura and R.A. Sunyaev, $A \& A$ A 24, 337 (1973).

5. A.F. Illarionov and R.A. Sunyaev, $A \mathscr{E} A$ 39, 185 (1975).

6. S. L. Shapiro and A.P. Lightman, ApJ 204, 555 (1976).

7. Y. Tanaka and W.H.G. Lewin, in X-ray Binaries, eds. W.H.G., J. van Paradijs, and E.P.J. van den Heuvel, 126 (Cambridge Univ. Press, Cambridge, 1995).

8. R. Hunt, MNRAS 154, 141 (1971).

9. J.M. Blondin, T.R. Kallman, B.A. Fryxell, and R.E. Taam, ApJ 356, 591 (1990). 
10. M. Ruffert, $A \mathscr{E} A$ 317, 793 (1997).

11. M. Ruffert, $A \mathscr{E} A$ 346, 861 (1999).

12. A.F. Illarionov and A.M. Beloborodov, MNRAS 323, 159 (2001).

13. S.L. Shapiro and S.A. Teukolsky, Black Holes, White Dwarfs, and Neutron Stars (Wiley-Interscience, New York, 1983).

14. J. Frank, A. King, and D. Raine, Accretion Power in Astrophysics (Cambridge Univ. Press, Cambridge, 1992).

15. Ya.B. Zel'dovich and N.I. Shakura, Soviet Astron. 13, 175 (1969).

16. J.P. Ostriker, R. McCray, R. Weaver, and A. Yahil, ApJ 208, L61 (1976).

17. A.F. Illarionov and D.A. Kompaneets, MNRAS 247, 219 (1990).

18. I.V. Igumenshchev, A.F. Illarionov, and D.A. Kompaneets, $M N R A S \mathbf{2 6 0}$, 727 (1993).

19. G.S. Bisnovatyi-Kogan, A.A. Ruzmaikin, Apध्GSS 28, 45 (1974).

20. P. Mészáros, A\&A 44, 59 (1975).

21. S.A. Balbus and J.F. Hawley, Rev. Mod. Phys. 70, 1 (1998).

22. M. Gierliński et al., MNRAS 288, 958 (1997).

23. M.L. McConnell et al., ApJ 424, 933 (1994).

24. M. Gierliński et al., MNRAS 309, 496 (1999).

25. P.S. Coppi, in High Energy Processes in Accreting Black Holes, eds. J. Poutanen and R. Svensson, ASP Conf. Series, 161, 375 (1999).

26. J. Poutanen, in Theory of Black Hole Accretion Disks, eds. M.A. Abramowicz, G. Björnsson, and J.E. Pringle, 100 (Cambridge Univ. Press, Cambridge, 1998).

27. A.A. Zdziarski, 1999, in High Energy Processes in Accreting Black Holes, eds. J. Poutanen and R. Svensson, ASP Conf. Series, 161, 16 (1999).

28. A.A. Galeev, R. Rosner, and G.S. Vaiana, ApJ 229, 318 (1979).

29. S.L. Shapiro, A.P. Lightman, and D.M. Eardley, ApJ 204, 187 (1976).

30. A.M. Beloborodov, in High Energy Processes in Accreting Black Holes, eds. J. Poutanen and R. Svensson, ASP Conf. Series, 161, 295 (1999).

31. A.M. Beloborodov and A.F. Illarionov, MNRAS 323, 167 (2001).

32. S.L. Shapiro and E.E. Salpeter, $A p J$ 198, 671 (1975).

33. I.V. Igumenshchev, A.F. Illarionov, and M.A. Abramowicz, ApJ 517, L55 (1999).

34. J. Wilms et al., MNRAS 320, 327 (2001). 